\title{
Guias de assistência à mulher com síndrome hipertensiva na Atenção Primária: revisão integrativa
}

\author{
Guides for assistance to women with hypertensive syndrome in Primary Care: integrative review \\ Directrices para la atención de mujeres con síndrome hipertensivo en Atención Primaria: revisión \\ integradora
}

Recebido: 15/02/2021 | Revisado: 21/02/2021 | Aceito: 24/02/2021 | Publicado: 02/03/2021

\author{
João Cruz Neto \\ ORCID: https://orcid.org/0000-0002-0972-2988 \\ Universidade Regional do Cariri, Brasil \\ E-mail: enfjcncruz@gmail.com \\ Paula Suene Pereira dos Santos \\ ORCID: https://orcid.org/0000-0001-6463-7316 \\ Universidade Regional do Cariri, Brasil \\ E-mail: paulasuene@yahoo.com.br \\ Emanuel Messias Silva Feitosa \\ ORCID: https://orcid.org/0000-0001-5278-3105 \\ Universidade Regional do Cariri, Brasil \\ E-mail: emfeitosa2017@gmail.com \\ Joseph Dimas de Oliveira \\ ORCID: https://orcid.org/0000 -0001-8105-4286 \\ Universidade Regional do Cariri, Brasil \\ E-mail: joseph.oliveira@urca.br
}

\begin{abstract}
Resumo
Objetivo: Identificar os guias de assistência à saúde da mulher com síndrome hipertensiva na atenção primária. Método: Trata-se de uma revisão integrativa da literatura que teve como questão norteadora: Quais os guias de assistência à saúde da mulher com síndrome hipertensiva na atenção primária?. Foi utilizada a estratégia PVO. A população foi composta por 4.518 estudos tendo como amostra cinco estudos. Resultados: Prevaleceram os guias derivados de instituições brasileiras que buscaram identificar as SHEG e definir um plano de ação para a morbidade. Contudo, permanece as abordagens medicamentosas em detrimentos de estratégias de promoção da saúde e prevenção de agravos. Foi identificada a hipertensão gestacional, a pré-eclâmpsia e a eclampsia como as principais comorbidades com a assistência direta prestada por médicos e enfermeiros. Conclusão: Destarte, evidencia-se uma prática baseada apenas na identificação e referênciamento da mulher sem o acompanhamento adequado, traçando-se as condutas apenas em fluxogramas. Ademais, destaca-se a necessidade de fomentar a prática baseada em evidências como método norteador dos cuidados no meio assistencial.
\end{abstract}

Palavras-chave: Complicações na gravidez; Hipertensão induzida pela gravidez; Atenção primária à saúde; Protocolos clínicos.

\begin{abstract}
Objective: To identify the guides of assistance to the health of women with hypertensive syndrome in primary care. Method: This is an integrative literature review with the following guiding question: What are the health care guides for women with hypertensive syndrome in primary care? The PVO strategy was used. The population was composed of 4,518 studies, with five studies as sample. Results: There was a prevalence of guides derived from Brazilian institutions that sought to identify SHEG and define an action plan for morbidity. However, drug approaches prevailed over strategies to promote health and prevent diseases. Gestational hypertension, pre-eclampsia and eclampsia were identified as the main comorbidities with direct assistance provided by physicians and nurses. Conclusão: Destarte-se evidencia-se uma prática baseada apenas na identificação e referenciamento da mulher sem o acompanhamento adequado, tracingando-se as condutas apenas em fluxogramas. Furthermore, there is a need to promote evidence-based practice as a method to guide care in the care assistence.
\end{abstract}

Keywords: Pregnancy complications; Pregnancy-induced hypertension; Primary health care; Clinical protocols.

\section{Resumen}

Objetivo: Identificar las guías de asistencia a la salud de la mujer con síndrome hipertensivo en la atención primaria. Método: Se trata de una revisión integradora de la literatura que tiene como pregunta principal: ¿Cuáles son las guías 
de asistencia a la salud de la mujer con síndrome de hipertensión en la atención primaria? Se utilizó la estrategia PVO. La población se compone de 4.518 estudios, con cinco estudios de referencia. Resultados: Hubo un predominio de guías derivadas de instituciones brasileñas que buscaban identificar el SHEG y definir un plan de acción para la morbilidad. Sin embargo, las estrategias de promoción de la salud y de prevención de los riesgos prevalecen sobre los enfoques farmacológicos. Se identificó la hipertensión gestacional, la preeclampsia y la eclampsia como las principales comorbilidades con la asistencia directa prestada por médicos y enfermeros. Conclusión: Se evidencia una práctica basada apenas en la identificación y referenciación de la mujer sin el acompañamiento adecuado, trazando las conductas apenas en fluxogramas. Además, destaca la necesidad de fomentar la práctica basada en las evidencias como método de mejora de los cuidados en el medio asistencial.

Palabras clave: Complicaciones del embarazo; Hipertensión inducida por el embarazo; Atención primaria; Protocolos clínicos.

\section{Introdução}

Os agravos em saúde no ciclo gravídico apresentam diferentes apresentações clinicas a depender da evolução e de implicações no potencial risco materno-fetal. Desta forma, as principais ocorrências atribuídas à Síndrome Hipertensiva Especifica da Gestação (SHEG) estão relacionadas a pré-eclâmpsia, eclampsia, hipertensão crônica, hipertensão gestacional e pré-eclâmpsia sobreposta a hipertensão crônica (Nóbrega, et al., 2016). Assim, as doenças hipertensivas e as hemorragias representam até $86 \%$ de todas as causas de morte materna no mundo (Kassebaum, et al.,2016; Oliveira Paixão, Fraga, Santos, \& Santos, 2017).

A hipertensão crônica (140 mmHg x $90 \mathrm{mmHg}$ ) está relacionada a presença da hipertensão anterior a gestação ou até a $20^{\mathrm{a}}$ semana gestacional. A Hipertensão gestacional está relacionada ao diagnóstico de hipertensão após a $20^{\mathrm{a}}$ semana de gestação. A pré-eclâmpsia se caracteriza pela hipertensão com lesão de órgão após as 20 semanas de gestação. A pré-eclâmpsia sobreposta a hipertensão crônica está relacionada a gestante hipertensa ou com enfermidades renais e hipertensão gestacional que cursa sem proteinúria; a eclampsia é identificada na presença de convulsões tônico cônicas ou coma em uma gestante que não tenha transtornos mentais ou outras patologias que levem a convulsão (Brito, et al., 2015; Auger, Fraser, Healy-Profitós, \& Arbour, 2015; Bezerra, Júnior, Feitosa, \& Carvalho, 2015).

A mulher no período gravídico tem como principal porta de entrada para o Sistema Único de Saúde a Atenção Primária a Saúde (APS), um sistema com acompanhamento integral, longitudinal e resolutivo com foco em prevenir e acompanhar a gestante em suas diferentes fases, com atividades que envolvem as manobras obstétricas, exame físico e sinais vitais (Neves, Flores-Quispe, Faccihini, Fassa, \& Tomasi, 2020).

É na APS que se concentra a assistência pré-natal como forma de prevenção de doenças e agravos em saúde. As consultas na gestação, esquema vacinal, uso do sulfato ferroso, rotina de exames complementares, exame físico e a completa anamnese são fatores importantes para o cuidado à gestante (Tomasi, et al., 2017). A assistência no período gestacional pode prever desfechos inesperados em até $85,7 \%$ dos casos, além de observar as desigualdades socioeconômicas e demográficas que dificultam o acesso aos serviços e ocasiona morbidades. Além do mais, é nesse tipo de atenção que se reduz o near miss materno e as complicações associadas aos distúrbios hemorrágicos e hipertensivos (Vidal, et al., 2016; Souza; Serinolli; \& Novaretti, 2019; Araújo, Santos, Santos, \& Frankin ,2017).

A importância do acompanhamento pré-natal está associada a assistência prestada à mulher em todo o período gestacional. De acordo com o Ministério da Saúde, as gestantes devem ser orientadas a realizar um número mínimo de seis consultas, incluindo exames específicos, imunização, suporte nutricional, identificação e tratamento de doenças base que podem agravar o quadro materno e conferir riscos. Essas consultas devem ser mensais até a $28^{a}$ semana, quinzenais entre 28 e 36 semanas e semanais até o término da gestação (Brasil, 2013).

O incentivo a formulação de políticas públicas para atender às demandas das causas hipertensivas e seus desfechos, deve subsidiar o cuidado integral em saúde. Nesse sentido, o auxílio nas estratégias de gestão em saúde deve contemplar o 
incentivo à produção de materiais que possam subsidiar profissionais engajados no planejamento e implementação de atividades que vislumbrem o cuidado em saúde (Mourão, Mendes, Marques, Cestari, \& Braga, 2019). Além do mais, a apropriação de tecnologias de assistência melhora a qualidade profissional e aumenta indicadores ligados ao controle de doenças (Pereira, Santos, Silva, \& Nagliate, 2017).

$\mathrm{Na}$ atenção primária são escassos os estudos que envolvem a utilização de ferramentas para identificação das síndromes hipertensivas. A partir do ano de 2000, observaram-se publicações acerca da assistência pré-natal das gestantes na atenção básica com um olhar voltado às condições básicas de saúde das grávidas (Thuler, Wall, Benedet, Sousa, \& Souza, 2018).

O serviço do enfermeiro no processo de parto e puerpério é primordial para a melhoria das condições de saúde. Por isso, é essencial resguardar a saúde do binômio mãe-feto, prevenir complicações e intervir a partir de alternativas que possam ofertar a melhor assistência com empatia, diminuindo situações que gerem ansiedade (Oliveira, et al., 2017; MartinezCastellón; Férandéz-Ordónes; \& Sánchez-Ruiz, 2018).

A orientação às gestantes é papel fundamental da atenção básica em saúde com intervenções que vão desde a prevenção de riscos e agravos, até os cuidados pós-parto. Quando não são fornecidas as orientações adequadas, as mulheres passam aos diferentes níveis de atenção reportando ansiedade e medo o que acarretam em desequilíbrio do estado geral e desfechos inesperados (Nóbrega, et al., 2016).

A continuidade da assistência e a particular atuação do enfermeiro nesse processo relaciona-se também a identificações dos fatores que podem ter implicações importantes na gestação. Nessa perspectiva, a consulta de enfermagem revela indicadores em saúde ao reconhecer os aspectos individuais, coletivos, comportamentais e familiares que influenciam desde hábitos alimentares, higiênicos, sexuais e sinais de bem-estar fetal além de preferências no parto e hospitalização até o ensino eficaz do aleitamento e cuidados puerperais (Oliveira, et al., 2017).

A identificação precoce dos sinais e sintomas de uma dada população perpassa a necessidade de direcionar o contexto em saúde alinhando a realidade com a utilização de alternativas que possibilitam o melhor desfecho clinico. Nesse sentido, a assistência de enfermagem torna-se essencial na previsão e identificação precoce das complicações pelas SHEG, com a possibilidade de padronização que permeia a utilização de instrumentos que norteiem aquilo que é essencial, observando a individualidade de cada paciente e adaptando as singularidades de cada realidade (Thuler, et al., 2018).

Os guias e manuais em revisões integrativas são estratégias que marcam uma estrutura teórica, com vistas a garantir uma sequência exata, exequível, precisa e confiável daquilo que já se tem produzido e que norteia um cuidado, desta forma, revisões neste sentido combinam diferentes métodos e avaliam a assistência que é prestada a um grupo ou comunidade (Synder,2019). Nesse sentido, objetivou-se identificar os guias de assistência à saúde da mulher com síndrome hipertensiva na atenção primária.

\section{Metodologia}

Trata-se de um estudo quantitativo do tipo revisão (Pereira, Shitsuka, Parreira, \& Shitsuka, 2018). Seguiu-se o método de integrativa, realizada por meio das etapas: identificação do tema, seleção da hipótese e definição da questão de pesquisa; estabelecimento de critérios para inclusão e exclusão de estudo/ amostragem ou busca e seleção na literatura dos estudos primários; definição das informações a serem extraídas dos estudos primários selecionados/categorização dos estudos; avaliação crítica dos estudos incluídos na revisão integrativa; análise e interpretação dos dados e síntese dos resultados ou apresentação da revisão (Mendes, Silveira, \& Galvão, 2019). 
Desta forma, a pesquisa teve início a partir da construção da questão norteadora:-"Quais os guias de assistência à saúde da mulher com síndrome hipertensiva na atenção primária?”. Optou-se pela estratégia Population, Variables and Outcomes (PVO) para a definição da população e do contexto, variáveis de interesse e de resultados (Bernardo, Nobre, \& Jatene, 2004) para busca dos artigos descritos no Quadro 1.

Quadro 1 - Descritores de assunto localizados no DeCS para os componentes da pergunta de pesquisa segunda a estratégia PVO. Crato, CE, Brasil, 2020.

\begin{tabular}{|l|l|l|l|}
\hline Itens da estratégia & Componentes & $\begin{array}{l}\text { Descritores do assunto } \\
\text { (DeCS) }\end{array}$ & $\begin{array}{l}\text { Descritores do } \\
\text { assunto (MeSH) }\end{array}$ \\
\hline Population & Gestantes & Complicações na gravidez & $\begin{array}{l}\text { Pregnancy } \\
\text { complications }\end{array}$ \\
\hline Variables & Assistência ao paciente & $\begin{array}{l}\text { Atenção primária à saúde; } \\
\text { Hipertensão induzida pela } \\
\text { gravidez }\end{array}$ & $\begin{array}{l}\text { Primary Health Care; } \\
\text { Hipertencion, } \\
\text { Pregnancy-induced }\end{array}$ \\
\hline Outcomes & Ferramentas de abordagem & Protocolos clínicos & Clinical Protocols \\
\hline
\end{tabular}

Fonte: Autores (2020).

A pesquisa foi realizada nas bases de dados: Literatura Latino-Americana e do Caribe em Ciências da Saúde (LILACS), Medical Literature Analysis and Retrieval System Online (MEDLINE) via EBSCO Information Services, Banco de dados em Enfermagem (BDENF), SCOPUS Preview, Web of Since e Cumulative Index to Nursing and Allied Health Literature (CINAHL). A busca foi realizada de forma pareada por pesquisadores distintos e realizada nos meses de março a abril de 2020 .

Para ambas as pesquisas se utilizou a estratégia de busca pregnancy complication AND primary health care AND hipertencion pregnancy-induced OR clinical protocols para as bases internacionais e complicações na gravidez AND atenção primária à saúde AND hipertensão induzida pela gravidez OR protocolos clínicos. Com os termos indexados nos descritores em ciências da saúde (DeCs) para bases latino-americanas e na Medical Subject Headings (MeSH) da PubMed para as de língua inglesa.

Os estudos foram submetidos a um processo de filtragem constituído pelos critérios de inclusão: guias e manuais disponíveis eletronicamente com texto completo online; publicados em português, inglês ou espanhol, de forma atemporal. Foram excluídos estudos primários, duplicados e a literatura cinzenta. Para a etapa de seleção dos estudos seguiu-se com a leitura dos títulos, resumos e a leitura integral dos textos para identificação daqueles que iriam compor o corpus da revisão.

Utilizou-se o formulário adaptado para coleta de dados proposto por Ursi \& Gavão (2006), para a organização das principais informações referentes aos estudos (Autor/Ano/País/ Objetivo/ Tipo de estudo/Nível de Evidência) e dados do desenvolvimento do artigo (Método e Conclusão).

A discussão dos estudos foi realizada de acordo com a classificação em sete níveis de evidência (NE) cientifica: I Evidências de revisões sistemáticas ou metanálise de ensaios clínicos; II - Evidência de pelo menos um ensaio clinico randomizado controlado bem desenhado; III - Estudos de ensaios clínicos sem randomização; IV - Estudos de coorte e caso controle com delineamento explicito; V - Revisão sistemática de estudos descritivos/qualitativos; VI- Que contemple um estudo qualitativo e VII - Opinião de autoridades/ relatórios de especialistas (Stilwell, Fineout-Overhalt, Melnyk, \& Willianson,2010). Os dados foram descritos em tabelas e interpretados com base na literatura pertinente.

Utilizou-se o instrumento Preferred Reporting Items for Systematic Rewiew and Meta-Analyses (PRISMA) para demostrar o processo de busca e seleção dos estudos (Fuchs \& Paim, 2010), conforme a Figura 1: 
Figura 1 - Fluxograma do processo de busca e seleção dos artigos por meio de cruzamentos. Crato, Ceará, 2020.
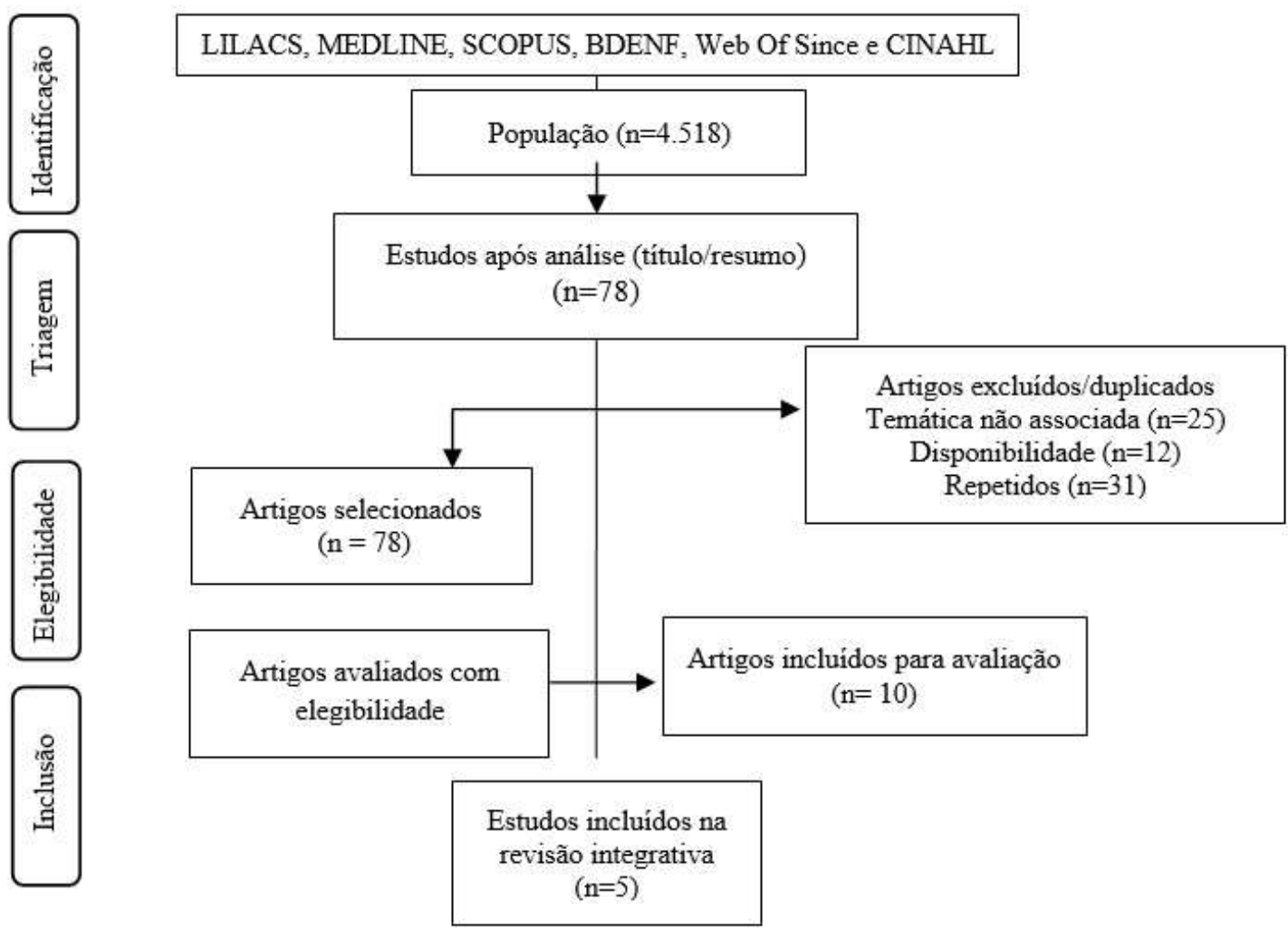

Fonte: Autores (2020).

\section{Resultados e Discussão}

A presente revisão incluiu cinco estudos (Quadro 2) analisados segundo autores, ano de publicação, país de publicação, objetivo dos estudos, metodologia utilizada e os principais resultados.

Dos estudos incluídos na presente revisão um foi publicado em 2011, dois em 2016, um em 2017 e um em 2019. Quanto ao nível de evidência foram identificados dois de nível III, dois níveis IV e um do nível I. Três estudos selecionados pertenciam ao Brasil, um a Argentina e um ao Perú. Dentre os estudos, foram identificados três estudos que reuniram as evidências cientificas, um era um guia de prática clínica e outro um protocolo clinico de obstetrícia. Quanto aos objetivos, os estudos abordaram recomendações para a padronização e orientação dos estados hipertensivos da gravidez, além de encaminhar a gestante ao serviço especializado. 
Quadro 2 - Caracterização dos estudos incluídos na revisão em relação aos componentes da pesquisa Crato, CE, Brasil, 2020.

\begin{tabular}{|c|c|c|c|}
\hline Autor/País & Objetivos & Guias/NE* & Resultados \\
\hline $\begin{array}{l}\text { 22.Marco et al } \\
(2011) \\
\text { Argentina }\end{array}$ & $\begin{array}{l}\text { Recomendações } \\
\text { para o cuidado } \\
\text { padronizado dos } \\
\text { estados } \\
\text { hipertensivos da } \\
\text { gravidez }\end{array}$ & 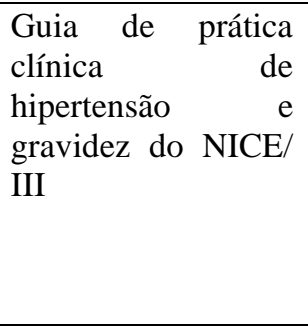 & $\begin{array}{l}\text { Hipertensão gestacional: Pressão arterial } \geq 140 / 90 \text {, } \\
\text { CIR }^{1} \text { e ILA }{ }^{2} \text {. Pré-eclâmpsia (PE): } \geq \text { a } 34 \text { semanas } \\
\text { finalização da gravidez. Hipertensão Crônica: } \\
\text { Labetolol, Nifedipina Metildopa. Hipertensão crônica } \\
\text { com pré-eclâmpsia sobreposta: Observa-se } \text { LOA }^{3} \text {. } \\
\text { Eclampsia: MgSO } 4 \text { gr/h por } 24 \mathrm{H} \text { e finalização da } \\
\text { gravidez. Síndrome Hellp: Dexametasona } 10 \mathrm{mg} \text { IV a } \\
\text { cada } 12 \mathrm{H} \text { durante } 48 \mathrm{H} \text { ) e hemoderivados. }\end{array}$ \\
\hline $\begin{array}{l}\text { 25.Harzheim, } \\
\text { Agostinho \& } \\
\text { Katz (2016) } \\
\text { /Brasil }\end{array}$ & $\begin{array}{l}\text { Determinar } \\
\text { encaminhamento } \\
\text { especializado }\end{array}$ & $\begin{array}{l}\text { Guia Clinico de } \\
\text { Obstetrícia/ IV }\end{array}$ & $\begin{array}{l}\text { Encaminhamento ao alto risco: } \mathrm{LOA}^{\mathrm{HAS}} \mathrm{HA}^{4}, \mathrm{DM}^{5} \text {, } \\
\text { uso de anti-hipertensivos e maior de } 40 \text { anos. } \\
\text { Hipertensão gestacional: Mal resultado obstétrico } \\
\text { e/ou perinatal em gestação prévia. Pré-eclâmpsia: } \\
\text { HAS, LOA e Proteinúria. Pré-eclâmpsia sobreposta a } \\
\text { hipertensão crônica: Sinal ou sintoma de PE. } \\
\text { Eclampsia: Crise convulsiva. }\end{array}$ \\
\hline $\begin{array}{l}\text { 27.Souza } \\
(2016) / \text { Brasil }\end{array}$ & $\begin{array}{ll}\text { Orientar } & \mathrm{o} \\
\text { atendimento } & \mathrm{a} \\
\text { gestante e ao feto } & \end{array}$ & $\begin{array}{l}\text { Guia com graus de } \\
\text { recomendação/ IV }\end{array}$ & $\begin{array}{l}\text { Proteger contra a prematuridade, manter perfusão } \\
\text { uteroplacentária, reduzir a hipóxia, crescimento } \\
\text { intrauterino restrito e óbito perinatal e, principalmente } \\
\text { a síndrome Hellp que causa a hemorragia cerebral. }\end{array}$ \\
\hline $\begin{array}{l}\text { 28.Enrique et } \\
\text { al (2017) /Peru }\end{array}$ & $\begin{array}{l}\text { Padronizar a prática } \\
\text { clinica }\end{array}$ & $\begin{array}{lr}\text { Guia } & \text { de } \\
\text { identificação } & \mathrm{e} \\
\text { encaminhamento } & \\
\text { das SHEG/I } & \end{array}$ & $\begin{array}{l}\text { O momento oportuno para iniciar o tratamento } \\
\text { Diurético osmótico é em pacientes com Eclampsia. A } \\
\text { importância do doppler uterino entre a semana } 11 \text { e } 14 \\
\text { prediz a pré-eclâmpsia com critérios de severidade e } \\
\text { aparição precoce. Administrar fluidoterapia em } \\
\text { gestantes com pré-eclâmpsia com critérios de } \\
\text { severidade a fim de evitar uma sobrecarga hídrica e } \\
\text { complicações secundárias. Existe repercussões no } \\
\text { neonato por uso de sulfato de magnésio em gestantes } \\
\text { com pré-eclâmpsia O dopper fetal é um marcador para } \\
\text { manejo expectante. Uso de AAS a partir da } 16^{a} \text { semana } \\
\text { de gestação. }\end{array}$ \\
\hline $\begin{array}{l}\text { 30.Tito et al } \\
\text { (2019) /Brasil }\end{array}$ & $\begin{array}{l}\text { Orientar } \\
\text { profissionais da } \\
\text { Atenção primária à } \\
\text { saúde. }\end{array}$ & $\begin{array}{l}\text { Guia para a clínica / } \\
\text { III }\end{array}$ & $\begin{array}{l}\text { Hipertensão crônica: com anti-hipertensivos envia ao } \\
\text { pré-natal de alto risco, níveis menores que } 140 / 90 \\
\text { mmHg, sem uso de anti-hipertensivo: } \\
\text { acompanhamento no centro de saúde, aferir pressão } 2 \text { a } \\
3 \text { vezes por semana sempre com avaliação. Deve-se } \\
\text { solicitar na primeira consulta, além de outros, } \\
\text { proteinúria de } 24 \text { horas, ECG }{ }^{6} \text { e creatinina. } \\
\text { Hipertensão gestacional: aferir } \text { PA }^{7} \text { diariamente, } \\
\text { consultas em } 7 \text { dias, orientar sobre sinais de alerta } \\
\text { (cefaleia, escotomas, epigastralgia, dor em hipocôndrio } \\
\text { e dispneia), realizar proteinúria de fita e exames } \\
\text { laboratoriais e cardiotocografia semanalmente. } \\
\text { Pré-eclâmpsia: Sem critérios de gravidade é } \\
\text { encaminhada ao pré-natal de alto risco com prioridade } \\
\text { e com critérios permanece internada até o parto. } \\
\text { Eclampsia: Encaminhamento a maternidade, manter } \\
\text { vias aéreas livre, oxigenação e puncionar acesso } \\
\text { venoso calibroso. } \\
\text { Pré-eclâmpsia sobreposta a hipertensão crônica: } \\
\text { Encaminhar a maternidade de referência para definição } \\
\text { do diagnóstico, com posterior internação. }\end{array}$ \\
\hline
\end{tabular}

Nota: *NE (Nível de Evidência), ${ }^{1}$ CIR (Crescimento intrauterino), ${ }^{2}$ ILA (Índice de líquido amniótico), ${ }^{3}$ LOA (Lesão de órgão alvo), ${ }^{4} \mathrm{HAS}$ (Hipertensão Arterial Sistêmica), ${ }^{5} \mathrm{DM}$ (Diabetes Mellitus), ${ }^{6} \mathrm{ECG}$ (Eletrocardiograma), ${ }^{7} \mathrm{PA}$ (Pressão arterial). Fonte: Autores (2020). 
Todos os estudos utilizaram-se de abordagens mistas ao incluir evidências quantitativas e qualitativas. Os principais profissionais envolvidos nos cuidados são médicos e enfermeiros ${ }^{(22-25-27-30)}$ ou somente médicos ${ }^{(28)}$. Os estudos direcionaram as recomendações para uma maternidade ${ }^{(22)}$ hospital universitário ${ }^{(25)}$, instituto materno perinatal ${ }^{(28)}$, prefeitura municipal ${ }^{(27-}$ ${ }^{30)}$, o sistema de saúde configura-se como misto em um pais ${ }^{(22)}$, segmentado ${ }^{(28)}$ e o SUS ${ }^{(25-27-30)}$.

A hipertensão gestacional (HG) foi assunto de três estudos ${ }^{(22-25-30)}$, dois estudos ${ }^{(25-30)}$ observam a HG como mal prognóstico se apresentado em até uma gestação anterior. Um estudo ${ }^{(30)}$, evidenciou a aferição da pressão arterial, exame laboratoriais e a cardiotopografia como meios de prevenção e controle de complicações.

A Pré-eclâmpsia foi observada em quatro estudos ${ }^{(22-25-28-30)}$, a finalização da gravidez é indicada se maior de 34 semanas ${ }^{(22)}$, quando acompanhado de Hipertensão Arterial Sistêmica (HAS), Lesão de órgão alvo (LOA) e proteinúria (25). Um estudo ${ }^{(28)}$ revelou que a administração de fluidoterapia e utilização do doppler fetal são marcadores seguros de reavaliação e condutas expectantes.

Quanto a HAS ${ }^{(22-30)}$, as recomendações são o uso de anti-hipertensivos como labetolol, nifedipina e metildopa além da realização dos exames de proteinúria de 24 horas, ECG e creatinina. Na pré-eclâmpsia sobreposta a hipertensão crônica ${ }^{(22-}$ ${ }^{25-30)}$, deve-se encaminhar ao alto risco e observar se existe LOA. Na eclampsia ${ }^{(22-25-28-30)}$, utiliza-se o MgSO4, diuréticos osmóticos, mantém vias aéreas livres e acesso calibroso. Para a síndrome Hellp preconiza-se o uso da dexametasona e a infusão de hemoderivados ${ }^{(27)}$. Os cuidados têm por finalidade proteger a prematuridade e o óbito fetal.

\section{Discussão}

O manejo terapêutico das síndromes hipertensivas passa por diferentes estratégias de cuidado como a administração de agentes anti-hipertensivos, anticonvulsivantes e ferramentas de gestão, para isso deve-se observar os sintomas referidos pelas mulheres tais como sintomas neurológicos, gastrointestinais e as demandas hipertensivas (González, et al., 2015). Esses dados corroboram com os principais sinais e sintomas apresentados pelas mulheres com SHEG.

Durante o estudo observou-se que o manejo clinico das síndromes hipertensivas traz o conceito e aplicação dentro da realidade onde se atualiza a evidência, bem como a identificação e os fatores relacionados a cada tema que leva em consideração a hipertensão crônica, hipertensão gestacional, pré-eclâmpsia sobreposta a hipertensão crônica, pré-eclâmpsia e eclampsia, outros estudos também ressaltam o correto manuseio e atualização com base nas patologias.

As condutas medicamentosas nas Síndromes Hipertensivas da Gravidez estão relacionadas, em parte, ao uso do ácido acetilsalicílico (AAS) (Thuler, et al, 2018). Quanto ao uso de AAS existe um poder antiplaquetário que reduz o risco de préeclâmpsia em mulheres com fatores de risco quando iniciado na decima sexta semana de gestação com uso de $75 \mathrm{mg} /$ dia (Lorente \& Rubio, 2016).

Duas estratégias importantes para a clínica da mulher com SHEG, relatada nos estudos, é a administração do sulfato de magnésio para tratamento da PE/E que possui algumas falhas tanto para administração quanto de materiais para suporte e manutenção da droga na APS. Outra alternativa é a administração do AAS, doppler fetal e uterino como medidas protetivas da pré-eclâmpsia.

Na assistência terciária o uso de sulfato de magnésio, hidralazina e metildopa estão associados a mulheres com fatores de risco entre os 19 e 35 anos, multíparas, maior de 38 semanas com cefaleia, escotomas e dor em epigastro e algumas alterações laboratoriais em leucócitos, hemoglobina, plaquetas, desidrogenasse lática, ácido úrico e proteinúria com desfecho na PE (Nóbrega, et al., 2016; González, et al., 2015). Contudo, quando isso se volta a atenção primária a infraestrutura e as condições de trabalho não permitem prestar esse tipo de assistência, como demostrado no estudo. 
Neste estudo, para o encaminhamento ao pré-natal de alto risco a mulher que é diagnosticada com alguma SHEG deve perceber, dentre os sinais e sintomas: hipertensão crônica ou gestacional com mal resultado obstétrico e/ou perinatal em gestação prévia; Pré-eclâmpsia alteração de PA, proteinúria, cefaleia persistente ou grave, anormalidades visuais, dor abdominal ou epigástrica, náusea e vômito, dispneia, dor retroesternal, confusão mental ou eclampsia que é a crise convulsiva em paciente com pré-eclâmpsia.

Mulheres com fatores de risco para doenças cardiovasculares e posteriormente as SHEG não possuem continuidade da assistência na APS o que dificulta o tratamento e a atenção pré-natal (Silva, et al., 2014). Além disso a prescrição de medicamentos e a manutenção dos mesmos é um fator que dificulta a autonomia do profissional e a prestação de cuidados (Martiniano, et al., 2016).

O processo de atenção em saúde e a utilização de recursos no setor depende da qualidade da assistência e denota um controle pré-natal eficaz ao reduzir os gastos diretos com a atenção em saúde (Brasil, 2016). Nesse sentido, necessita-se de uma rede de apoio pautada na referência e contra referência para mulheres com SHEG (Silva, et al., 2014).

Quanto a gravidade das doenças hipertensivas, o corpo torna-se um ambiente teratogênico e que destrói as células trofoblasticas, o excesso de fatores vasculares leva ao dano tecidual com exacerbação do prognóstico clinico, essas alterações podem ser passiveis de tratamento quando corretamente acompanhadas pela atenção primária (Alonso-Remédios, PérezCutiño, \& Delgado, 2017). Quando há uma incorreta gestão do cuidado na APS os outros níveis de atenção tornam-se superlotados, o que reflete na baixa qualidade dos atendimentos (Ekawati, et al., 2017).

A rede de referência e contra referência não se dá de forma vertical nos cuidados, mas se estende pela horizontalidade das ações e prevê o acompanhamento da atenção primária mesmo quando a gestante é direcionada a outros níveis de complexidade, quando não existe essa abordagem mesmo com número mínimo de consultas realizadas o risco de desenvolver complicações continua alto (Nóbrega, et al.,2016).

Destaca-se que a SHEG é a principal causa de morte materna, e esse tipo de morte pode ser considerado como um importante indicador de desenvolvimento humano visto que há uma necessidade de políticas públicas, que viabilizem o cuidado qualificado da gestante na rede de atenção melhorando sua qualidade de vida (Araújo, et al., 2017).

Ressalta-se ainda nesse estudo que o manejo da hipertensão arterial visa proteger contra a prematuridade, manter perfusão uteroplacentária, reduzir a hipóxia, crescimento intrauterino restrito e óbito perinatal e, principalmente a síndrome Hellp que causa a hemorragia cerebral.

A atuação do enfermeiro e de uma equipe multiprofissional é imprescindível não só na tomada de decisões como na redução da morte materna e do seu concepto, iniciando com práticas de educação em saúde, ainda na APS (Mariano et al., 2018). A sensibilização da gestante e a adoção de comportamentos para um estilo saudável possibilita a mudanças nas situações de vida e nas crenças, exigindo do profissional um dinamismo nas atividades de forma criativa e interativa alinhando as necessidades adstritas da população gestante e o incentivo ao autocuidado (Thuler, et al., 2018).

Existe pouca adesão a protocolos nos sistemas em saúde principalmente na APS, ao constituir uma visão integrada do cliente essas medidas não farmacológicas devem estar interligadas a um olhar multiprofissional em que para sua implementação necessita de gestores, profissionais e indivíduos que tem por objetivo a redução dos riscos maternos e a continuidade da assistência (Dantas, et al, 2018).

\section{Conclusão}

O cuidado à gestante com síndrome hipertensiva deve ser ponto norteador da assistência do pré-natal perpassando todos os níveis de atenção. 
Observa-se nos estudos a prática baseada apenas na identificação e referênciamento da mulher sem o acompanhamento adequado, ainda na atenção primária, traçando as condutas apenas em fluxogramas. O reconhecimento dos sinais e sintomas, que variam conforme a SHEG apresentada pode ser encontrada nos protocolos, além da terapia farmacológica implementada.

O manejo clinico das síndromes hipertensivas depende do curso prognóstico das patologias com destaque a préeclâmpsia e eclampsia com e sem critérios de gravidade e na presença de lesão de órgão alvo, encaminhando ao alto risco quando observado hipertensão crônica ou gestacional, pré-eclâmpsia e associação com sintomas neurológicos e gastrointestinais.

Os estudos com gestantes acometidas pelas síndromes hipertensivas na atenção primária à saúde revelam a necessidade de melhores investimentos no setor e principalmente, quanto a estratégias de gestão e ferramentas de gestão clínica, gerenciamento de fluxo e sistematização do cuidado a esse público especifico além de terapia oral medicamentosa.

Ressalta-se como limitações a pouca amostra encontrada e fomenta-se o desenvolvimento de novas pesquisas que possam elucidar as formas de cuidado a gestante, com a melhor evidência cientifica, e aplicado no contexto da atenção primária.

\section{Referências}

Alonso-Remedios, A., Perez-Cutino, M. L. D., \& Dania, F. (2017). Inmunopatogenia de la enfermedad hipertensiva gravídica. Rev Cubana Obstet Ginecol, 43 (4):102-114.

Araújo, I. F. M., Santos, P. A., Santos, P. A., \& Frankin, T. A. (2017). Síndrome hipertensivas e fatores de risco associados à gestação. Rev.Enferm UFPE online, Recife,4254-62.

Araújo, M. A. L., Andrade, R. F. V., Barros, V. L., \& Bertonceli, P. M. R. P. (2019). Fatores associados aos desfechos desfavoráveis provocados pela Sífilis na gestação. Rev. Bras. Saúde Mater. Infant, 19(2)421-429.

Auger, N., Fraser, W. D., Healy-Profitós, J., \& Arbour, L. (2015). Association Between Preeclampsia and Congenital Heart Defects. Jama, 314(15)1588-1598.

Bernardo, W. M, Nobre, M. R. C, \& Jatene, F. B. (2004). A Prática Clínica Basead A Em Evidências. Parte II - Buscando As Evidências Em Fontes De Informação. Rev. Assoc Med Bras, 50(1)104-8.

Bezerra, E. H., Júnior, C. A., Feitosa, R. F., \& Carvalho, A. A. (2015). Mortalidade materna por hipertensão: índice e análise de suas características em uma maternidade-escola. Rev Bras Ginecol Obstet. 27(9): 548-53.

Brasil. (2013). Ministério da Saúde. Secretaria de Atenção à Saúde. Departamento de Atenção Básica. Atenção ao pré-natal de baixo risco. Editora do Ministério da Saúde.

Brasil. (2016). Sintese de evidências para politicas de saúde: Mortalidade perinatal. Ministério da Saúde, Secretaria de Ciência, Tecnologia e Insumos Estratégicos, Departamento de Ciência e Tecnologia. (2a ed.), Ministério da Saúde, p.40.

Brito, K. K. G., Moura, J. R. P., Sousa, M. J., \& Brito, J. V., Oliveira, S. H. S., Soares, M. J. G. O. (2015). Prevalência das síndromes hipertensivas especificas da gestação (SHEG). Rev. J.res: fundam. Care. Online, 7(3):2717-2725.

Dantas, R. C. O., Dantas, D. C. O., Lima, V. V., Silva, J. P. T., Amador, A. E., Lopes, M. S., Azevedo, U. M., \& Roncalli, A. G. (2018). O Uso De Protocolos Na Gestão Do Cuidado Da Hipertensão Arterial Na Atenção Primária À Saúde: Uma Revisão Integrativa. Revista Ciência Plural, 4(1):117-31.

Ekawati, F. M., Licqurish, S., Emilia, O., Gunn, J., Brennecke, S., \& Lau, P. (2017). Developing management pathways for hypertensive disorders of pregnancy (HDP) in Indonesian primary care: a study protocol. Reproductive Health, 16(1):1-10.

Enrique, G. R, Francisco, P. A. C., Walter, D. P. M., Antonio, L. R. O., Alfonso, M. S. L., Liliana, C. G. S., Elizabeth, R. A. M, Marleny, H. B, Erasmo, T. A., Yahira, O. S. J., Oswaldo, G. C., Antonio, L. F., Eloisa, I. R. C., Santiago, C., Delfina, A. R. N., Victor, S. M., \& Emilia, M. M. C. (2017). Guia de prática clínica para a prevenção e manejo da pré-eclâmpsia e eclampsia. Série guias de prática clinica $\mathrm{n}^{\circ} 2$.

Fuchs, S. C, \& Paim, B. S. (2010). Revisão Sistemática De Estudos Observacionais Com Metanálise. Rev HCPA, 30(3):294-301, 2010.

González, A. I. I., Fajardo, J. J. C., Alvarado, J. A. V, Rodríguez, N. I. M., Rivas, O. J. D., Mejia, R. J. T., Rivas, O. O. D., Serrano, R. D. F, \& Urbia, M. C. B. (2016). Trastornos Hipertensivos Del Embarazo: Clínica Y Epidemiología, Hospital Regional Santa Teresa 2015. Rev Med Hondur. 84(3):101-106.

Harzheim, E., Agostinho, M. R., \& Katz, N. Protocolos de encaminhamento para obstetrícia (Pré-Natal de alto risco). Atendimento para médicos e enfermeiros da APS/AB do Brasil. Disponível em: www.telessauders.ufrgs.br. Acesso em: 23/04/2020. 
Kassebaum, N. J., Barber, R. M., A Bhutta, Z., Dandona, L., Gething, P. W, Hay, S. I, Kinfu, Y., Larson, H. J., Liang, X., \& Lim, S. S. (2016). Global, regional, and national levels of maternal mortality, 1990-2015: a systematic analysis for the global burden of disease study 2015.The Lancet, 388(10053):1775-1812.

Lorente, M. A., \& Rubio, G. N. (2016). Prevención de la preeclampsia com aspirina. Rev. Prog. Obstet. Ginecol. 59(3):180-184.

Marco, I. D., Basualdo, M. N., Pietrantonio, E. D., Paladino, S., Ingilde, M., Domergue, G., \& Velarde, C. n. Guía De Práctica Clínica: Estados hipertensivos del embarazo 2010. (2011). Revista del Hospital Materno Infantil Ramón Sardá, 30(2):70-93.

Mariano, M. S. M., Belarmino, A. C., Vasconcelos, J. M. S., Holanda, L. C. A., Siqueira, D. D., \& Junior, A. R. F. (2018). Mulheres com síndromes hipertensivas. Rev enferm UFPE on line, Recife, 12(6):1618-24.

Martinez-Castellón, N. Fernández-Ódónez, E, \& Sánchez-Ruiz, P. Manejo del síndrome de Hellp em uma gestación pretérmino. Rev. Matronas Prof, 19(2):1720 .

Martiniano, C. S., Coêlho, A. A., Souza, M. B., Brandão, I. C. A., Silva, A. K. F., \& Uchôa, S. A. C. (2016). Caracterização da prescrição de medicamentos por enfermeiros nos protocolos de Atenção Primária à Saúde. Revista Enfermagem Uerj, 24(3): 1-7.

Mendes, K. D. S., Silveira, R. C. C. P., \& Galvão, C. M. (2019). Uso de gerenciador de referências bibliográficas na seleção dos estudos primários em revisão integrativa. Texto Contexto Enferm, 28(1):1-15.

Mourão, L. F., Mendes, I. C, Marques, A. D. B., Cestari, V. R. F, \& Braga, R. M. B. B. (2019). Internações em UTI por causas obstétricas. Rev. Enfermeris global, 53:318-31.

Neves, R. G, Flores-Quispe, M. D. P, Faccihini, L. A, Fassa, A. G., \& Tomasi, E. (2020). Pré-natal no Brasil: estudo transversal do Programa de Melhoria do Acesso e da Qualidade da Atenção Básica, 2014. Rev. Epidemiol. Serv. Saude, 29(1):1-12.

Nóbrega, M. F, Santos, M. T. B. R., Davim, R. M. B, Oliveira, L. F. M., Alves, E. S. R. C., \& Rodrigues, E. S. R. C. (2016). Perfil de gestantes com síndrome hipertensiva em uma maternidade pública. Rev. Enferm UFPE online, 10(5):1805-11.

Oliveira, G. S., Paixão, G. P. N., Fraga, C. D. S., Santos, M. K. R., \& Santos, M. A. (2017). Assistência de enfermeiros na síndrome hipertensiva gestacional em hospital de baixo risco obstétrico. Rev. Cuidarte, 8(2):1561-72.

Pereira, A. S., Shitsuka, D. M., Parreira, F. J., \& Shitsuka, R. (2018). Metodologia da pesquisa científica.

Pereira, G. T., Santos, A. A. P., Silva, J. M. O., \& Nagliate, P. C. (2017). Perfil epidemiológico da mortalidade materna por hipertensão: análise situacional de um estado nordestino entre 2004-2013. Re. Fund care online, 9(3):653-658.

Silva, M. L. C., Galvão, A. C. A. A., Souza, N. L., Azevedo, G. D., Jerônimo, S. M. B., \& Araújo, A. C. P. F. (2014). Women with cardiovascular risk after preeclampsia: is there follow-up within the unified health system in Brazil? Revista Latino-americana de Enfermagem, 22(1):93-99.

Snyder, H. Literature review as a research methodology: an overview and guidelines. Journal Of Business Research, 104(1):333-339.

Souza, E. C. (org). (2016). Manual do cuidado no pré-natal e puerpério na atenção primária à saúde. Prefeitura Municipal de Londrina, PR. Londrina, 2ed, p.1-434.

Souza, I. A., Serinolli, M. I., \& Novaretti, M. C. Z. Prenatal and puerperal care and indicators of severity: a study about the information available on pregnant women's card. Revista Brasileira de Saúde Materno Infantil, 19(4):983-989.

Stilwell, S. B., Fineout-Overhalt, E., Melnyk, B. M., \& Willianson, K. M. (2010). Evidence-based practice: step by step. AJW, 110(5):41-47.

Thuler, A. C. M. C., Wall, M. L., Benedet, D. C. F., Sousa, S. R. R. K., \& Souza, M. A. R. (2018). Medidas preventivas das sindromes hipertensivas da gravidez na atenção primária. Rev. Enferm UFPE on line, 12(4):1060-71.

Tito, A. D. C. (org). (2019). Protocolo pré-natal e puerpério. Prefeitura de Belo Horizonte. $2^{\mathrm{a} e d .}$

Tomasi, E., Fernandes, P. A. A., Fischer, T., Siqueira, F. C. V., Silveira, D. S., Thumé, E., Duro, S. M. S., Saes, M. O., Nunes, B. P., \& Fassa, A. G. (2017). Qualidade da atenção pré-natal na rede básica de saúde do Brasil: indicadores e desigualdades sociais. Cadernos de Saúde Pública, 33(3):1-11.

Ursi, E. S, \& Galvão, C. M. (2006). Prevenção De Lesões De Pele No Perioperatório: Revisão Integrativa Da Literatura. Rev. Latino-Am Enfermagem, 14(1):124-31.

Vidal, C. E. L., Carvalho, M. A. B., Grimaldi, I. R., Reis, M. C., Baêta, M. C. N., Garcia, R. B., Silva, S. A. R. (2016). Morbidade materna grave na microrregião de Barbacena/MG. Cadernos Saúde Coletiva, 24(2):131-138. 